\title{
Balloon Laryngoplasty for Pediatric Subglottic Stenosis: A 5-year Experience
}

\author{
Original Investigation $>\begin{aligned} & \text { Serap Şahin Önder }{ }^{1} \text { DD, Aslı Şahin Yılmaz }{ }^{1} \text { (D), Özgül Gergin² }{ }^{1} \text { De, Begüm Y1lmaz }{ }^{1} \text { (D) } \\ & \text { Department of Otolaryngology, University of Health Sciences, Ümraniye Training and Research Hospital, İstanbul, Turkey }\end{aligned}$ \\ ${ }^{2}$ Department of Otolaryngology, Medipol University Hospital, İstanbul, Turkey
}

\begin{abstract}
Objective: The objective of this study was to present our experience and evaluate our results of endoscopic balloon laryngoplasty (BL) in children with subglottic stenosis (SGS) at a pediatric tertiary center over a 5-year period.

Methods: This study reviewed 41 pediatric patients diagnosed with acquired SGS who had undergone $\mathrm{BL}$ as the primary course of treatment. Cases were analyzed for details including patient demographics, SGS grade and length, timing and the type of surgery, presence of tracheostomy, comorbidities, postoperative management, complications and outcomes of balloon dilatation.

Results: Forty-one children (22 girls and 19 boys) who had undergone $\mathrm{BL}$ at a mean age of 26 months (range, 1 month to 14 years) were included in the
\end{abstract}

study. Nineteen $(46.3 \%)$ were diagnosed with acute SGS (12 thin stenosis, 7 thick stenosis) and 22 (53.7\%) with chronic SGS (9 thin stenosis, 13 thick stenosis). The success rate of BL was $100 \%$ in patients with acute and chronic thin membranous stenosis. The effectiveness of BL was significantly higher in patients with acute thick stenosis than in patients with chronic thick stenosis ( $\mathrm{p}=0.016$ ).

Conclusion: This study confirms that $\mathrm{BL}$ in patients presenting with acquired SGS with thin membranous stenosis, regardless of whether acute or chronic, can have a good prognosis. However, the results are less promising in cases of chronic thick stenosis.

Keywords: Subglottic stenosis, balloon laryngoplasty, airway, pediatric, pediatric otolaryngology
ORCID iDs of the authors: S.S. $0.0000-0002-3576-0953$; A.S.Y. 0000-0002-7846-9453; 0.G. 0000-0002-4544-863X; B.Y. 0000-0002-1345-2503.

Cite this article as: Sahin ÖnderS, Şahin-YImaz A, Gergin Ö, YIImaz B. Balloon Laryngoplasty for Pediatric Subglottic

Stenosis: A 5-year Experience. Turk Arch Otorhinolaryngol 2020; 58(4): 208-13.

\section{Corresponding Author:}

Serap Şahin Önder; serapsahinonder@gmail.com

Received Date: 19.07.2020

Accepted Date: 25.08 .2020

Content of this journal is licensed under a Creative Commons Attribution 4.0 International License. Available online at www.turkarchotolaryngol.net

\section{Introduction}

Subglottic stenosis (SGS) encompasses a range of potentially life-threatening conditions and is characterized by the narrowing of the airway below the vocal folds. SGS has an incidence reporting of 1 to $8 \%$ after endotracheal intubation (1). Advancements in neonatal care coupled with the introduction of prolonged intubation methods in neonatal and pediatric intensive care units have markedly reduced mortality rates in critically ill children over the last 60 years. Consequently, there is an increase in the incidence of SGS due to the application of mechanical ventilation $(2,3)$.

The treatment of SGS should be patient-centric with the utmost importance placed on all practices that aid in delivering successful surgeries. This involves a number of factors including the type of the stenosis, the surgeon's experience as well as the preferred course of treatment, expectations of the patient and the family, the quality of post-operative care the institution can provide, and the skill level of the caregivers (anesthesiologist, pulmonologist and gastroenterologist) (4).

The surgical techniques deployed fall into two groups, as endoscopic and open procedures. La- ryngotracheal reconstruction, cricotracheal resection and cervical slide tracheoplasty feature prominently in the rankings for the frequently used open airway reconstruction procedures (3). However, the last 5-20 years, has seen the increased use of endoscopic approaches, especially after the introduction of the balloon dilator (4). Although balloon laryngoplasty (BL) has been used since the 1980s, it remains a relatively new technique in the treatment of SGS and is now heralded as an effective and less invasive treatment for appropriate stenosis cases (5).

The objective of this study was to present our experience and evaluate our results of endoscopic BL in children with SGS at a pediatric tertiary center over a 5 -year period.

\section{Methods}

This is a retrospective review of all patients who presented with SGS, were diagnosed and referred to our tertiary care hospital between 2015 and 2020. Patient charts were analyzed and reviewed for data points, including patient demographics, grade of subglottic stenosis (Cotton-Myers classification (6)), timing (acute or chronic SGS; Avelino et al. (7)), craniocaudal extension of SGS 

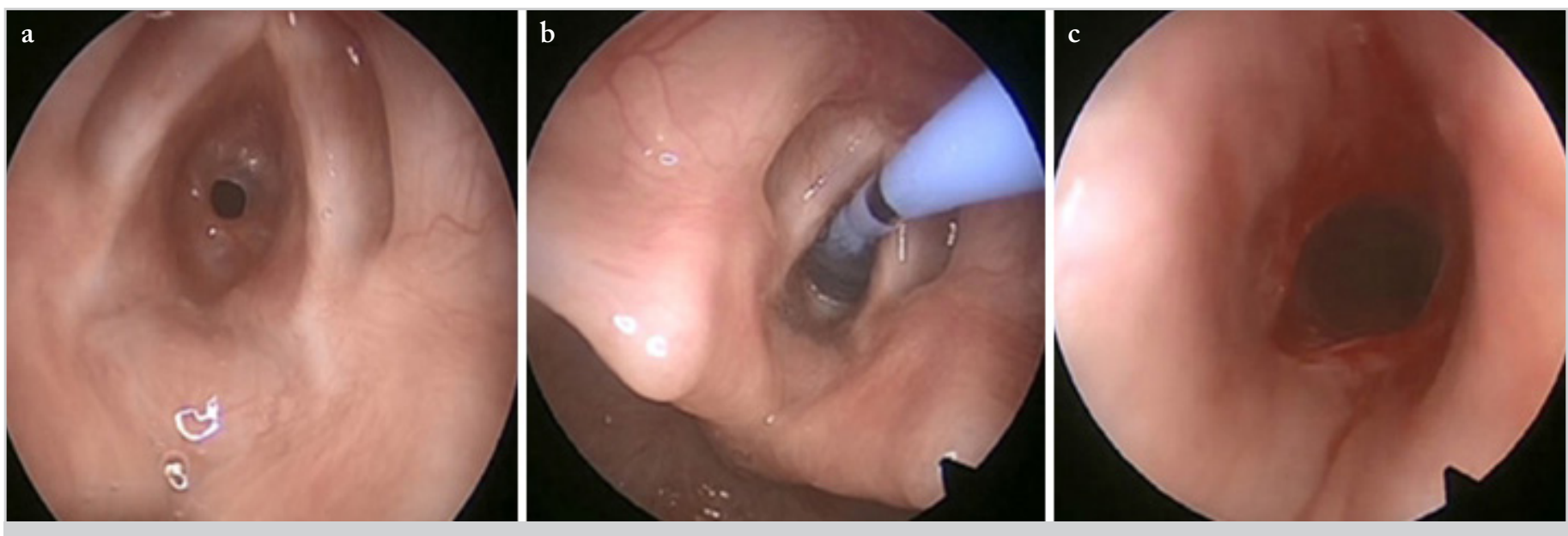

Figure 1. a-c. Acute grade III thin SGS in a 3-month old child. (a) pre-balloon laryngoplasty, (b) with balloon laryngoplasty, (c) post-balloon laryngoplasty

(thin or thick; McCaffrey classification (8)), type of surgery, presence of tracheotomy, comorbidities, postoperative management, complications, as well as the following outcomes: change in endoscopic grading of SGS, postoperative symptoms, rate of decannulation, tracheotomy status. Informed consent was taken from the parents. Local Ethics Committee of Ümraniye Training and Research Hospital approved the study (Approval Date: March 19, 2020; Approval Number: 7507).

This series included 41 pediatric patients with documented SGS who had undergone endoscopic BL as a primary modality of treatment. Patients who had other coexisting tracheal pathologic conditions, grade 4 SGS, SGS with glotto-subglottic stenosis, congenital SGS, previous intervention for their SGS, comorbidities requiring a high likelihood of prolonged ventilation in the future or lost to follow-up after treatment were excluded. The diagnosis and description of subglottic stenosis was performed by an expert pediatric otolaryngologist at the time of the direct laryngobronchoscopy (DLB) with spontaneous ventilation technique. The Myer-Cotton grade was used to assess the severity of SGS (6).

The timings of SGS were classified as follows.

1) Acute SGS was defined as a diagnosis of SGS within 30 days of extubation,

2) Chronic SGS was defined as a diagnosis of SGS 30 days after extubation (7).

The lengths of the scar tissue were classified as follows.

1) Thin SGS, which has a craniocaudal extension of $<1 \mathrm{~cm}$ (McCaffrey classification grade 1)

\section{Main Points}

- $\quad$ SGS is not a rare complication for the newborns or infants with prolonged intubation for various reasons in neonatal and pediatric intensive care units.

- $\quad$ SGS is a life-threatening condition.

- $\mathrm{BL}$ is a less invasive treatment for SGS.

- BL can have a good prognosis in patients with acquired SGS with thin stenosis regardless of whether acute or chronic.
2) Thick SGS, which has a craniocaudal extension of more than $1 \mathrm{~cm}$ and features either soft or hard scar contraction (McCaffrey classification grades 2 and 3) (8).

Prior to BL, dynamic upper airway examination was performed under spontaneous respiration using a flexible bronchoscope (3.1 mm or $3.8 \mathrm{~mm}$ Olympus Medical Systems; Hamburg, Germany) in order to exclude the presence of possible additional airway diseases including laryngomalacia, vocal cord paralysis or tracheomalacia. DLB was performed under general anesthesia using a $0^{\circ}$ rigid endoscope (Storz Corporation; Tuttlingen, Germany) with a diameter between 2.7 and $4 \mathrm{~mm}$. After establishing the diagnosis, high-pressure balloon (Acclarent Technology; Boston, United States) was used and inflation pressure of 8-12 atm was maintained for 1 to 2 minutes or until the oxygen saturation level dropped below $90 \%$. The balloon size was selected according to the theoretical ideal subglottic diameter for the patient's age (9). The procedure was performed three times each session. A pledget soaked with betamethasone dipropionate and gentamycin sulfate (Belogent; Farmatek, İstanbul, Turkey) was then applied to the dilated segment. Adjunctive procedure, such as radial divisions of stenosis was performed using cold knife in thick SGS featuring hard scar contraction. No patient was intubated after dilatation; instead, monitored in the pediatric intensive care unit for 24 to 48 hours. Follow-up endoscopy was performed after an interval of three weeks in all cases, and BL was performed when required. All patients received systemic steroids (Prednisolone; Actavis, İstanbul, Turkey) (1-2 mg/kg/d) for two days, proton pump inhibitors (Ulcuran; Avis, İstanbul, Turkey) for one month postoperatively. Follow-up DLB was performed three weeks after the initial DLB and the procedure was repeated until complete healing. After complete resolution of SGS, the children underwent a course of awake flexible fiberoptic laryngoscopy (FFL) every three months for a year. If the results were not satisfactory, BL was performed three times at 3-week intervals. If this failed to yield positive results, open surgery was performed. All DLB and awake FFL examinations were recorded (Figures 1, 2, 3, 4). Only when patients had been weaned from tracheotomy or had become symptom free for the duration of the study were deemed successful BL outcomes. The 

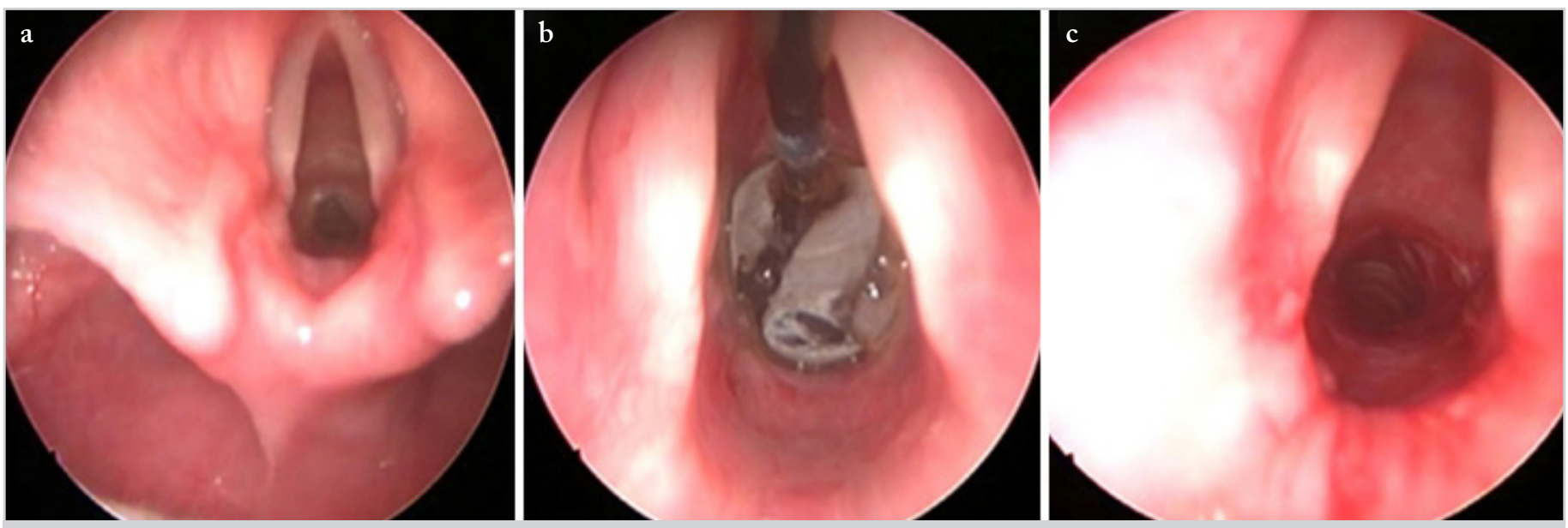

Figure 2. a-c. Acute grade II thick subglottic and tracheal stenosis in an 11-year-old child. (a) subglottic view of the lumen, (b) with balloon laryngoplasty, (c) post-balloon laryngoplasty
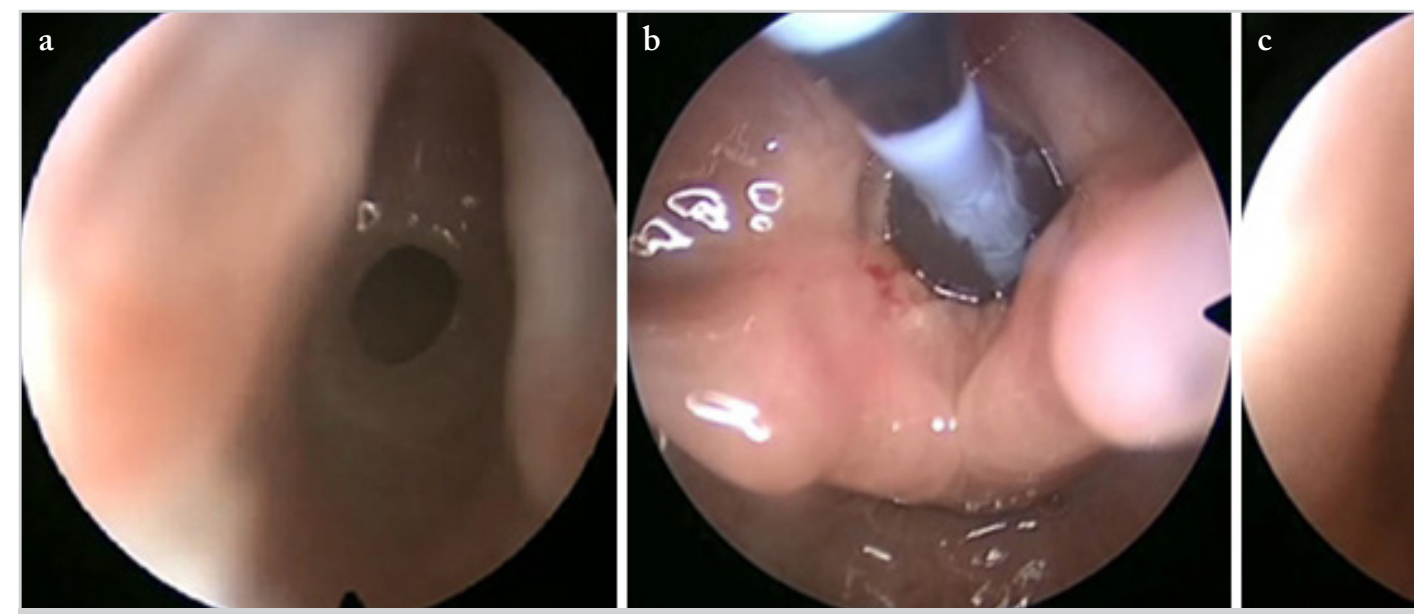

Figure 3. a-c. Chronic grade II thin SGS in a 7-month old child. (a) subglottic view of the lumen, (b) with balloon laryngoplasty, (c) post-balloon laryngoplasty
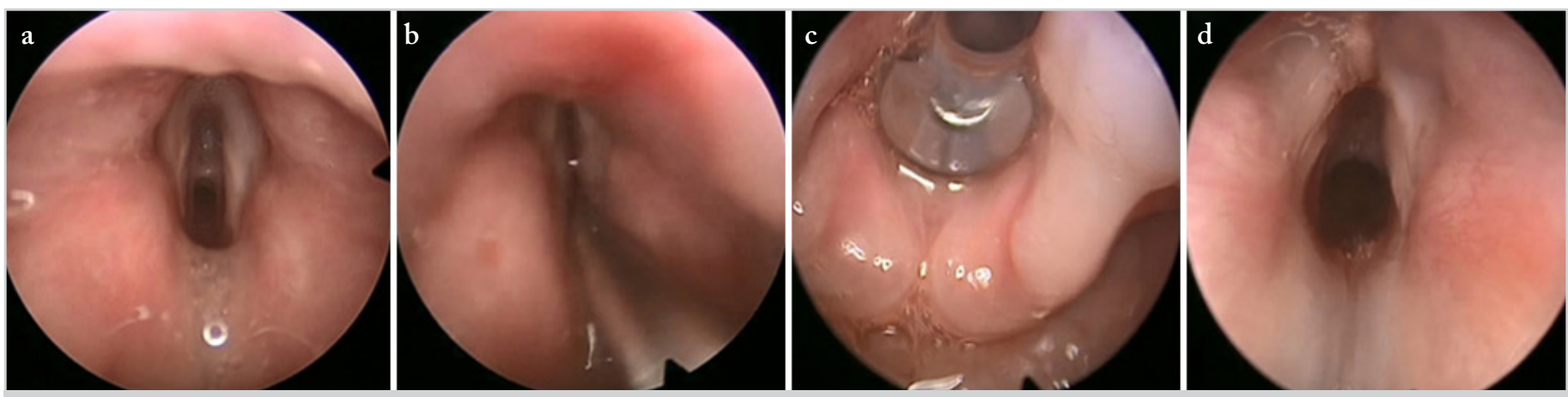

Figure 4. a-d. Chronic grade III thick SGS in a 22-month old child (a) subglottic view of the lumen, (b) radial divisions of stenosis using cold knife, (c) with balloon laryngoplasty, (d) post-balloon laryngoplasty

effectiveness of BL was calculated according to the changes in the Cotton-Myer grade with measurements taken before and after balloon dilatation.

\section{Statistical Analysis}

IBM Statistical Package for the Social Sciences version 20.0 (IBM SPSS Corp.; Armonk, NY, USA) was used for statistical analysis. The changes in the Cotton-Myer grades before and after the BL were compared using the Mann-Whitney U Test. $\mathrm{P}$-values of $\leq 0.05$ were regarded as significant.

\section{Results}

Forty-one children (22 girls and 19 boys) who had undergone $\mathrm{BL}$, at a mean age of 26 months (range, one month to 14 years) were included in the study. Table 1 summarizes the breakdown of the patients by demographics, comorbidities, and details of 
the etiology of stenosis. Nineteen (46.3\%) children were diagnosed with acute SGS (12 thin stenosis, seven thick stenosis) and 22 (53.7\%) with chronic SGS (nine thin stenosis, 13 thick stenosis). Thirteen patients had undergone a tracheotomy before

Table 1. Demographics, comorbidities, etiology and follow up Patients $(n=41)$

\begin{tabular}{l|c}
\hline Age range & Patients (n=41) \\
\hline Gender & $\begin{array}{c}\text { (month-14 years } \\
\text { mean: } 26 \text { months) }\end{array}$ \\
\hline Male & \\
\hline Female & $19(46.3 \%)$ \\
\hline Comorbidity & $22(53.7 \%)$ \\
\hline Prematurity & $11(26.8 \%)$ \\
\hline Only CHD & $5(12.1 \%)$ \\
\hline Down syndrome + CHD & $3(7.3 \%)$ \\
\hline CEA & $1(2.4 \%)$ \\
\hline Type 1 DM & $1(2.4 \%)$ \\
\hline None & $20(48.7 \%)$ \\
\hline Etiology of SGS & \\
\hline Prolonged intubation & $41(100 \%)$ \\
\hline Mean follow up period & $15 \pm 8.6$ months \\
\hline CHD: congenital heart disease; CEA: congenital esophageal atresia; DM: diabetes \\
mellitus; SGS: subglottic stenosis
\end{tabular}

laryngeal stenosis assessment. The mean period of follow-up was $15 \pm 8.6$ months. The etiology pointed to all patients having developed SGS due to prolonged intubation. Other associated medical comorbidities were shown in Table 1.

The details of the children regarding their SGS grade and other features were shown in Table 2. In patients with acute and chronic thin stenosis, the number of BLs ranged from one to two times. Moreover, the mean numbers of BLs in acute and chronic thin SGS groups were $1.3 \pm 0.7$ and $1.2 \pm 0.4$, respectively. However, in acute and chronic thick SGS groups this range was from two to three times. The mean numbers of BLs in acute and chronic thick SGS groups were $2.4 \pm 0.7$ and $2.3 \pm 0.7$, respectively. In all patients, BL was performed at 3-week intervals. The success rate of BL was $100 \%$ in patients with acute and chronic thin stenosis. In the acute thick SGS group $(\mathrm{n}=7)$ six children had undergone tracheotomies before BL and successful decannulation was achieved in two patients after balloon laryngoplasty. Three patients required additional open airway surgeries and the success rate of $\mathrm{BL}$ was four out of seven (57.1\%). In the chronic thick SGS group $(n=13)$ tracheotomy was performed in seven children before BL and one case was successfully decannulated after balloon laryngoplasty. Nine patients required additional open airway surgeries. In this group, the success rate of BL was four out of 13 (30.7\%) (Table 2).

There was no significant statistical difference between the effectiveness of $\mathrm{BL}$ in patients with acute and chronic thin stenosis $(p=0.56)$. BL was significantly more effective in patients with thin SGS (acute or chronic) than in patients with thick SGS (acute or chronic) $(\mathrm{p}=0.000)$. Moreover, the effectiveness of $\mathrm{BL}$

Table 2. Comparison of outcomes between acute and chronic SGS

\begin{tabular}{|c|c|c|c|c|}
\hline \multirow{2}{*}{$\begin{array}{l}\text { Type of SGS } \\
\text { Length of stenosis }\end{array}$} & \multicolumn{2}{|c|}{ Acute (n=19) } & \multicolumn{2}{|c|}{ Chronic (n=22) } \\
\hline & Thin stenosis $(\mathrm{n}=12)$ & Thick stenosis $(\mathrm{n}=7)$ & Thin stenosis $(n=9)$ & Thick stenosis $(n=13)$ \\
\hline \multicolumn{5}{|l|}{ Pre-op grade* } \\
\hline Grade I & - & - & $11.1 \%(\mathrm{n}=1)$ & $7.6 \%(\mathrm{n}=1)$ \\
\hline Grade II & $8.3 \%(\mathrm{n}=1)$ & $57.1 \%(\mathrm{n}=4)$ & $33.3 \%(n=3)$ & $53.8 \%(\mathrm{n}=7)$ \\
\hline Grade III & $91.7 \%(\mathrm{n}=11)$ & $42.9 \%(n=3)$ & $55.5 \%(\mathrm{n}=5)$ & $38.4 \%(\mathrm{n}=5)$ \\
\hline \multicolumn{5}{|l|}{ Post-op grade* } \\
\hline Grade 0 & $100 \%(n=12)$ & $28.5 \%(n=2)$ & $100 \%(\mathrm{n}=9)$ & - \\
\hline Grade I & - & $42.8 \%(n=3)$ & - & $23.1 \%(n=3)$ \\
\hline Grade II & - & $14.2 \%(\mathrm{n}=1)$ & - & $53.8 \%(\mathrm{n}=7)$ \\
\hline Grade III & - & $14.2 \%(n=1)$ & - & $23.1 \%(n=3)$ \\
\hline Number of BL & $1.3 \pm 0.7$ & $2.4 \pm 0.7$ & $1.2 \pm 0.4$ & $2.3 \pm 0.75$ \\
\hline Success rate of $\mathrm{BL}$ & $100 \%$ & $57.1 \%$ & $100 \%$ & $30.7 \%$ \\
\hline Presence of tracheotomy & N/A & $85.7 \%(n=6)$ & None & $53.8 \%(\mathrm{n}=7)$ \\
\hline Decannulation rate after $\mathrm{BL}$ & N/A & $33.3 \%(\mathrm{n}=2)$ & None & $14.2 \%(\mathrm{n}=1)$ \\
\hline
\end{tabular}


Şahin Önder et al.

was significantly higher in patients with acute thick stenosis than in patients with chronic thick stenosis $(\mathrm{p}=0.016)$.

There were no significant clinical complications related to the procedures.

\section{Discussion}

Airway narrowing in children is mostly located in the subglottis. The most expected pathogenesis of acquired SGS commences with subglottic mucosal pressure necrosis due to endotracheal intubation, then mucosal ulceration, followed by perichondritis, and finally mature scar tissue formation (10). This condition necessitates immediate treatment on symptom presentation. The purpose of $\mathrm{BL}$ is to arrest the progress of mature scar formation during the evolution of acquired SGS.

The lack of prospective studies evaluating the effectiveness of balloon dilatation has come to mean that balloon dilatation is predominantly featured in discussions. Designing controlled studies comparing $\mathrm{BL}$ to other more conventional therapies is challenging due to patients having a wide range of comorbidities and lack of uniformity amongst airway patients (e.g., age, weight, gender, ethnicity, stenosis diameter, length, histology, neurologic functions, glottal status and comorbidities) (11). Consequently, case series are an important mechanism for experience sharing.

The presented case series included 41 pediatric patients with acquired SGS who had undergone BL. The subglottic stenosis cases were divided into four subgroups: acute thin stenosis, acute thick stenosis, chronic thin stenosis, and chronic thick stenosis. Our results revealed that the effectiveness of BL may vary depending on the type of the subglottic stenosis. This study adhered to the guidelines established by the previous reporting by McCaffrey (8) for the purposes of this case series, however, it should be stressed that SGS measurements can be subjective.

The management of SGS in children is a frustrating and distressing process both for the patients and their families. In acute cases, it is possible that several extubation attempts are unsuccessful or extubation is successful but the child ends up with severe respiratory distress. In chronic cases, the child has already had a tracheotomy or is receiving treatment for recurring laryngitis or asthma. According to several studies, the success rate of BL for acute SGS was significantly higher compared to chronic SGS $(2,7)$. Avelino et al. (7) demonstrated a success rate of $100 \%$ for acute SGS and $39 \%$ for chronic SGS and determined that success rates could be linked to the SGS grade and the immature scar tissue. Lee et al. (2) suggested that patients with acquired SGS, once diagnosed, should promptly undergo balloon dilatation as it increases treatment success rates, and decannulation as well, to reduce the need of tracheotomies. However, Simpson et al. (12) warned that in patients with chronic acquired SGS, the prognosis could be worse if circumferential stenosis and scar contraction are present. In the presented study, balloon dilatation demonstrated a $100 \%$ success rate in patients with SGS with thin and soft membrane, regardless of whether the scar tissue was acute or chronic. This success rate was $57.1 \%$ in patients with acute thick stenosis and $30.4 \%$ in patients with chronic thick stenosis. These results reaffirmed that when the stenosis features thin and soft membranous scar tissue, regardless of whether acute or chronic, the success of balloon dilatation is virtually certain. If the stenosis is fibrotic and thick in character, prompt execution of balloon dilatation is paramount to ensure success, as the stenosis expands easier during the acute period than in the chronic period. Therefore, early diagnosis and treatment of acquired SGS is vital to prevent recurrence. Children who have undergone prolonged endotracheal intubation and develop stridor and respiratory distress after extubation need extra consideration and care.

On reviewing the literature, we found that one systematic review, which included 7 studies and 150 patients, estimated the overall success rate of $\mathrm{BL}$ as $65.3 \%$. The authors claimed that failures may be linked to more severe grades of stenosis (13). In our series, the success rate was calculated by ignoring the types of stenosis and scar tissue. The presented study demonstated that where the grade of SGS is severe, the success of balloon dilatation is high in the patients with acute and chronic thin SGS. Moreover, this outcome shows that the nature of stenosis is more important than its grade.

There are varying numbers of dilatations reported in the literature. Some authors describe performing up to seven dilatations until achieving success, but most report that up to three dilatations were necessary for successful treatments (14). In our study, serial DLB was performed as previously described and deemed successful if there were any responses in the form of grading, extension, or clinical picture. If there was no improvement in the patient's condition after three consecutive sessions, we moved to open surgical procedures. For each patient with acute or chronic thin SGS, the number of BLs performed ranged from one to two times. In patients with acute and chronic thick SGS, however, this number ranged from two to three times. Unfortunately, the outcomes of three patients in the acute thick SGS group and nine patients in the chronic thick SGS group were not satisfactory.

Special attention should be devoted to balloon dilatation in children who have not undergone tracheotomy. Close collaboration with the anesthesiology team is vital in pediatric airway surgeries (15). Reported complications related to balloon dilatation of the lower airway include granuloma formation, mucosal laceration, tracheitis, bleeding, pneumothorax, pneumomediastinitis, atelectasis, and death (15). Furthermore, a rapid, complete luminal obstruction secondary to edema in response to balloon dilatation was reported by Gungor (16). This complication can cause an inability to intubate. All surgeons need to be alert about the presence of this complication should it arise, the occurrence of which would necessitate an emergency tracheotomy. The downside risks posed by BL can be managed by reviewing the location, the size of the stenosis, and by analyzing the patient's underlying health condition (15). In our study, none of the patients experienced any major complications from BL. 


\section{Conclusion}

This study highlights the evidence that $\mathrm{BL}$ in pediatric patients with acquired thin SGS, regardless of whether of acute or chronic, can have a good prognosis. BL may be considered as a first-course treatment for thin acquired SGS. However, further research is advocated for its use in the management of SGS especially with thick and mature stenosis.

Ethics Committee Approval: Ethics committee approval was received for this study from the Local Ethics Committee of Ümraniye Training and Research Hospital (Approval Date: March 19, 2020; Approval Number: 7507).

Informed Consent: Informed consent was obtained from the parents of the patients who participated in this study.

Peer-review: Externally peer-reviewed.

Author Contributions: Concept - S.Ş.Ö., Ö.G.; Design - S.Ş.Ö., Ö.G.; Supervision - A.Ş.Y., Ö.G.; Resources: Ö.G.; Materials - S.Ş.Ö., B.Y.; Data Collection and/or Processing - S.Ş.Ö., B.Y.; Analysis and/or Interpretation - S.Ş.Ö., B.Y.; Literature Search - S.Ş.Ö., A.Ş.Y., B.Y.; Writing - S.Ş.Ö., A.Ş.Y.; Critical Reviews - S.Ş.Ö., A.Ş.Y.

Conflict of Interest: The authors have no conflicts of interest to declare.

Financial Disclosure: The authors declared that this study has received no financial support.

\section{References}

1. Morrissey MS, Bailey CM. Diagnosis and management of subglottic stenosis after neonatal ventilation. Arch Dis Child 1990; 65: 1103-4.

2. Lee JC, Kim MS, Kim DJ, Park DH, Lee IW, Roh HJ, et al. Subglottic stenosis in children: our experience at a pediatric tertiary center for 8 years in South Korea. Int J Pediatr Otorhinolaryngol 2019; 121: 64-7.

3. Marston AP, White DR. Subglottic stenosis. Clin Perinatol 2018; 45: 787-804.
4. Smith MM, Cotton RT. Diagnosis and management of laryngotracheal stenosis. Expert Rev Respir Med 2018; 12: 709-17.

5. Avelino MG, Fernandes EJ. Balloon laryngoplasty for subglottic stenosis caused by orotracheal intubation at a tertiary care pediatric hospital. Int Arch Otorhinolaryngol 2014; 18: 39-42.

6. Myer CM 3rd, O'Connor DM, Cotton RT. Proposed grading system for subglottic stenosis based on endotracheal tube sizes. Ann Otol Rhinol Laryngol 1994; 103: 319-23.

7. Avelino M, Maunsell R, Jubé Wastowski I. Predicting outcomes of balloon laryngoplasty in children with subglottic stenosis. Int J Pediatr Otorhinolaryngol 2015; 79: 532-6.

8. McCaffrey TV. Classification of laryngotracheal stenosis. Laryngoscope 1992; 102: 1335-40.

9. Hautefort C, Teissier N, Viala P, Van Den Abbeele T. Balloon dilation laryngoplasty for subglottic stenosis in children: eight years' experience. Arch Otolaryngol Head Neck Surg 2012; 138: 235-40.

10. Baker S, Kelchner L, Weinrich B, Lee L, Willging P, Cotton R, et al. Pediatric laryngotracheal stenosis and airway reconstruction: a review of voice outcomes, assessment, and treatment issues. J Voice 2006; 20: 631-41.

11. Bent JP, Shah MB, Nord R, Parikh SR. Balloon dilation for recurrent stenosis after pediatric laryngotracheoplasty. Ann Otol Rhinol Laryngol 2010; 119: 619-27.

12. Simpson GT, Strong MS, Healy GB, Shapshay SM, Vaughan CW. Predictive factors of success or failure in the endoscopic management of laryngeal and tracheal stenosis. Ann Otol Rhinol Laryngol 1982; 91: 384-8.

13. Wentzel JL, Ahmad SM, Discolo CM, Gillespie MB, Dobbie AM, White DR. Balloon laryngoplasty for pediatric laryngeal stenosis: case series and systematic review. Laryngoscope 2014; 124 : 1707-12.

14. Rutter MJ, Cohen AP, de Alarcon A. Endoscopic airway management in children. Curr Opin Otolaryngol Head Neck Surg 2008; 16: 525-9.

15. Lee KH, Ko GY, Song HY, Shim TS, Kim WS. Benign tracheobronchial stenoses: long-term clinical experience with balloon dilation. J Vasc Interv Radiol 2002; 13: 909-14.

16. Gungor A. Balloon dilation of the pediatric airway: potential for disaster. Am J Otolaryngol 2012; 33: 147-9. 